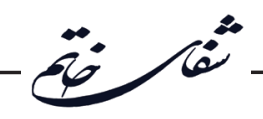

\title{
Investigation of Dysfunction in the Neural Systems Underlying Social Perception, Action Observation and Theory of Mind in Autism Spectrum Disorders
}

\section{Chiman Saeedi*}

Psychologist, Kurdistan, Iran

\section{A BSTRACT}

Introduction: Autism spectrum disorders (ASD) are neurodevelopmental disorders that are characterized by difficulties in social communication and social interaction as well as by restricted and repetitive behaviors, interests or activities. Researches within the field of social neuroscience demonstrate that social functioning deficits in ASD may arise from dysfunctions in the neural systems that implicate in social information processing. In this study, existing research related to the neural basis of three key neural systems underlying social information processing (social perception, action observation, and theory of mind and malfunction of neural systems) in ASD have been reviewed. The results indicate that there is atypical activation in brain regions involved in social perception (the posterior superior temporal sulcus (PSTS), the amygdala, the orbitofrontal cortex, and the fusiform gyrus), in regions associated with action observation consist of mirror neurons system and its three interconnected areas (PSTS, the inferior frontal gyrus, and the inferior parietal lobule), and in regions assign to theory of mind (the medial prefrontal cortex, the tempro-parietal junction, PSTS, the posterior cingulate cortex/precuneus, and the anterior temporal lobes). Conclusion: These results indicate aberrant social information processing in ASD and define it as deficits in the neural systems involved in social perception, action understanding, and theory of mind. These findings provide a foundation for understanding the neural mechanisms underlying social deficits in ASD and highlight the role of PSTS as a common region in all three systems.

\section{Key words:}

1. Autism Spectrum Disorder

2. Social Perception

3. Theory of Mind

*Corresponding Author: Chiman Saeedi

E-mail:Ch.Saeedi@Yahoo.com 
بررسى اختلال در سيستمهاى عصبى اساسى ادراك اجتماعى، مشاهدهُ عمل و نظرية ذهن در اختلالات طيف اوتيسم ادراعتماعم

\section{"جيمن سعيدى "}

روانشناس، كردستان، ايران

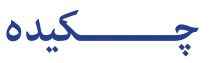

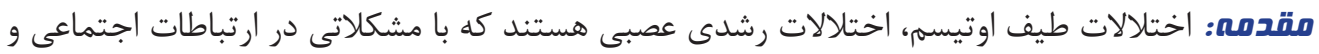

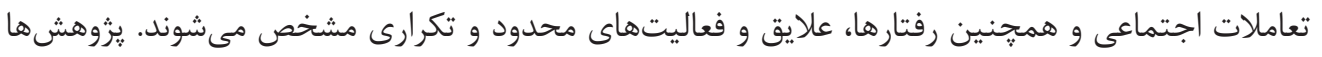

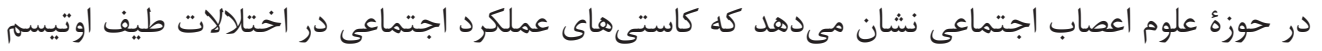

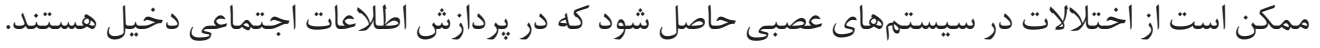

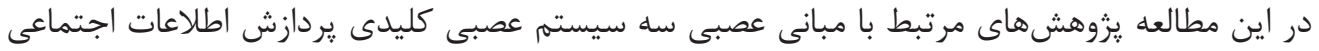

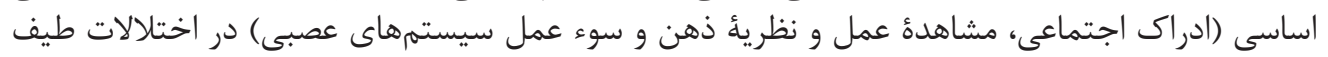

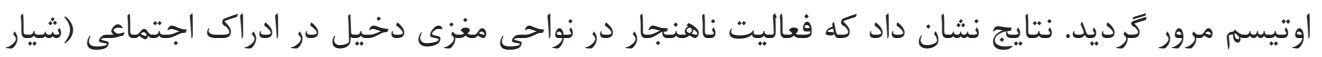

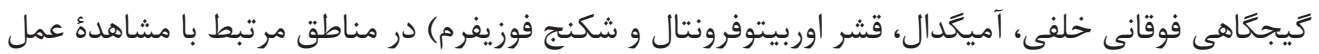

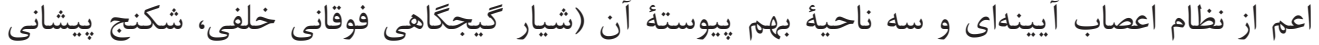

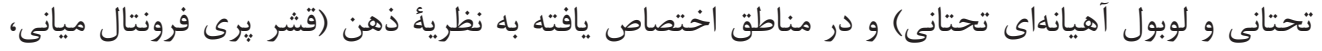

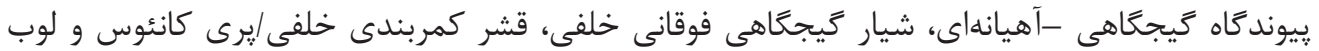

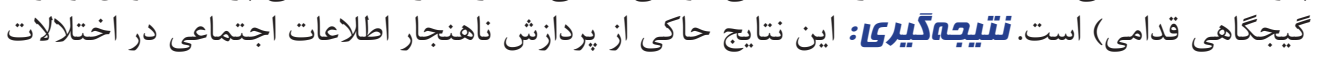

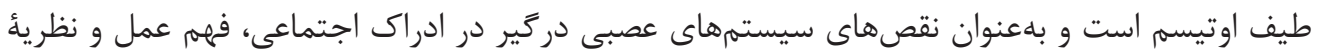

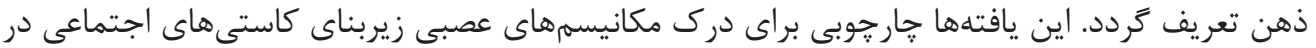

كليد وازهها:

1. اختلالات طيف اوتيسم

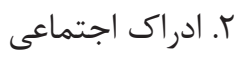

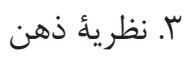

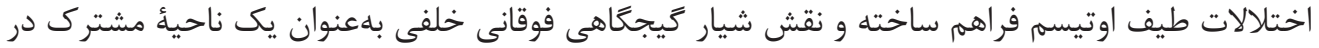

$$
\text { هر سه سيستم برجسته مى الات طسازد. }
$$


محركهـــاى اجتماعـى حســاس هسـتند (I ). نقــش كليــدى در شـــكئه ادراك اجتماعـى ايفــاء

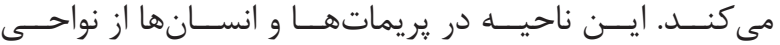

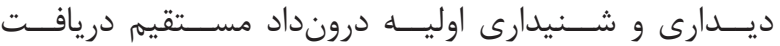

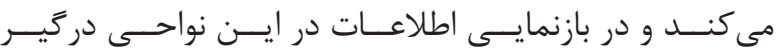

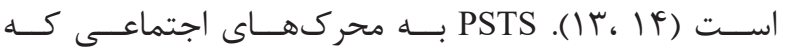

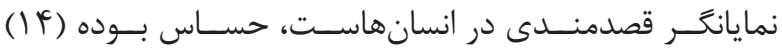

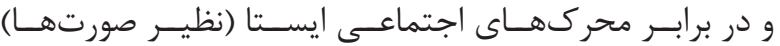

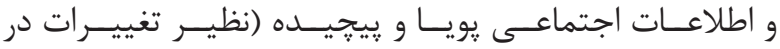

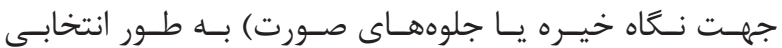

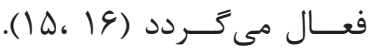

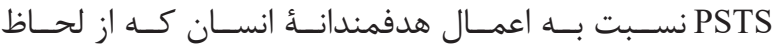

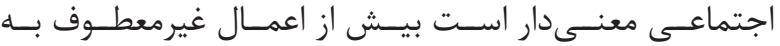

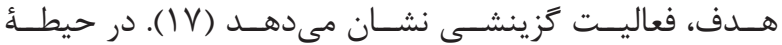

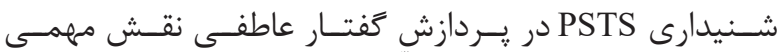

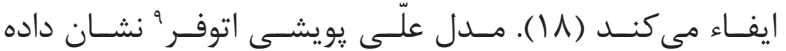

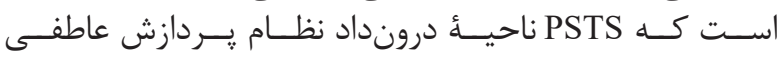

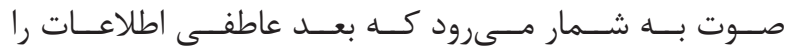
اســتخراج و بازنمايسى مى كنـــــ (19 ().

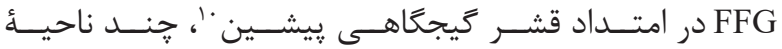

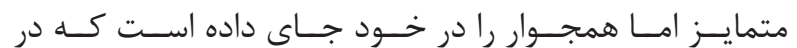

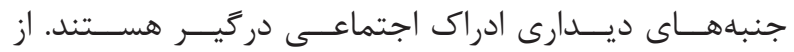

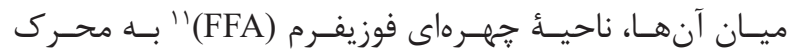

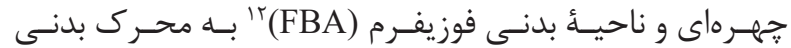

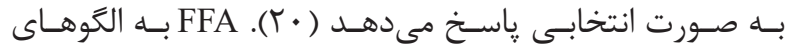

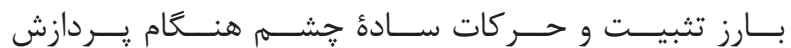

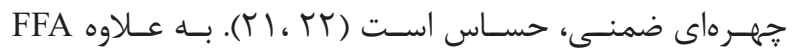

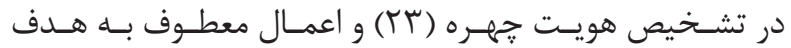

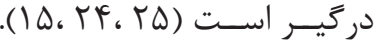

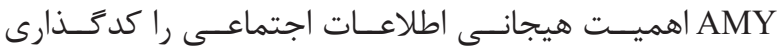

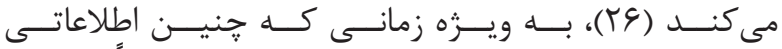

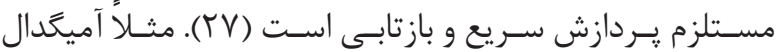

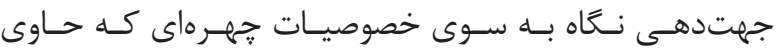

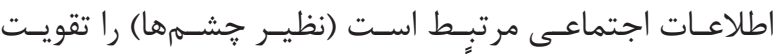

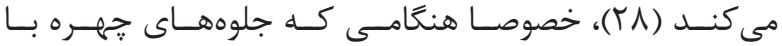

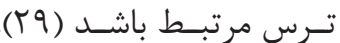

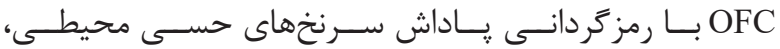

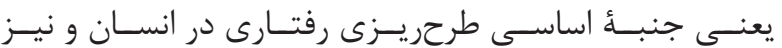

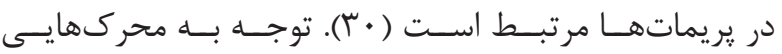

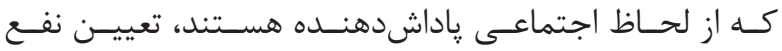

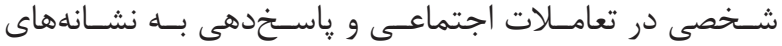

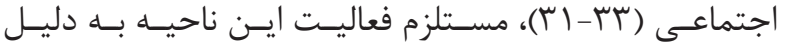

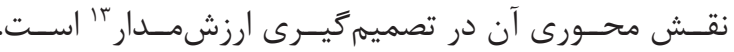

${ }^{1}$ Diagnostic and statistical manual of mental disorders

${ }^{2}$ Autism spectrum disorder

${ }^{3}$ Social perception

${ }^{4}$ Rhesus monkeys

${ }^{5}$ Posterior superior temporal sulcus

${ }^{6}$ Amygdala (AMY)

${ }^{7}$ Orbitofrontal cortex

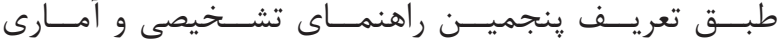

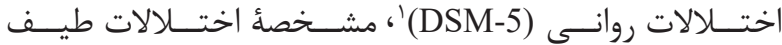

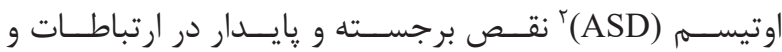

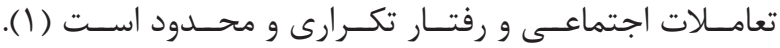

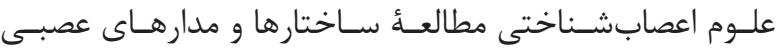

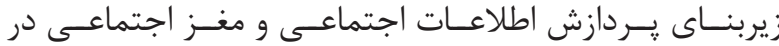

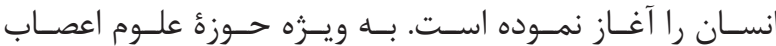

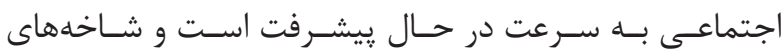

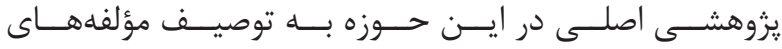

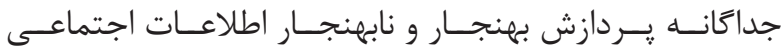

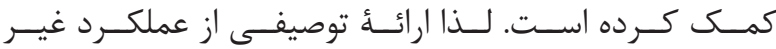

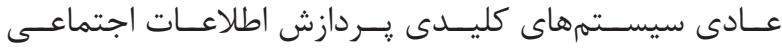

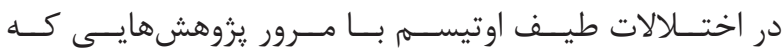

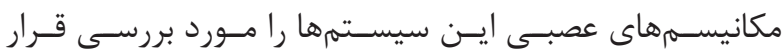

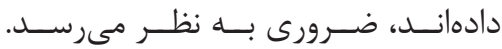
سيستم عصبى ادراك اجتماعى

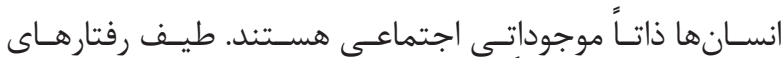

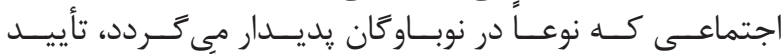

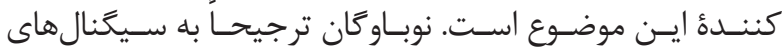

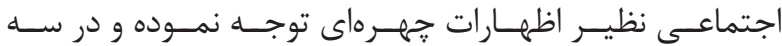

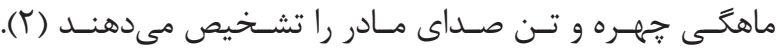

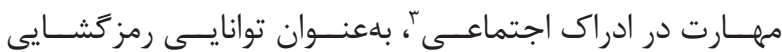

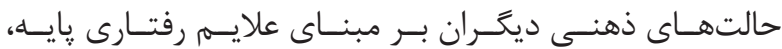

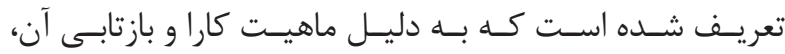

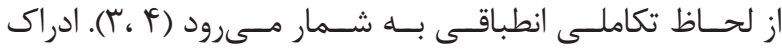

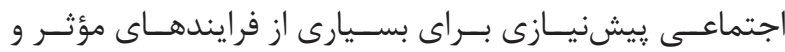

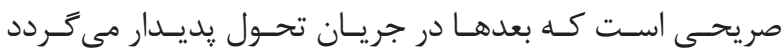

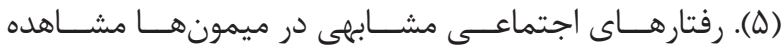

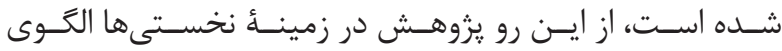

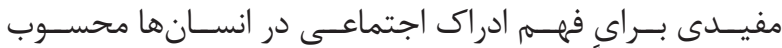

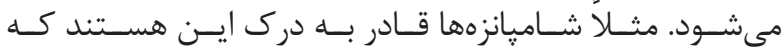

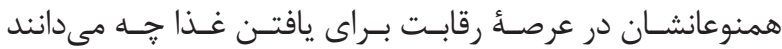

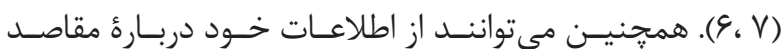

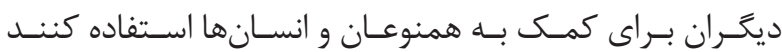

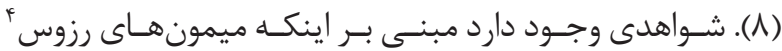

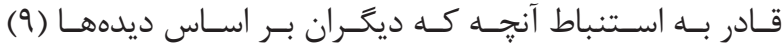

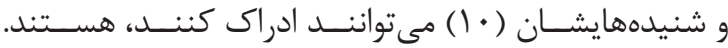

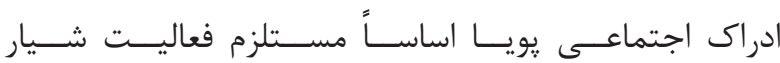

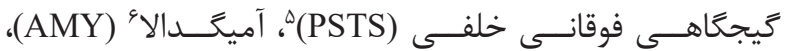

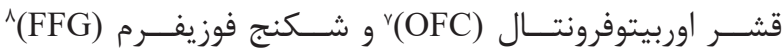

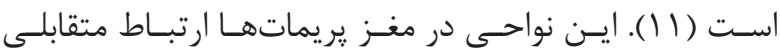

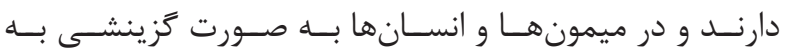

${ }^{8}$ Fusiform gyrus

${ }^{9}$ Ethofer dynamic causal modeling

${ }^{10}$ Ventral-temporal cortex

${ }^{11}$ Fusiform face area

${ }^{12}$ Fusiform body area

${ }^{13}$ Value-based decision-making 


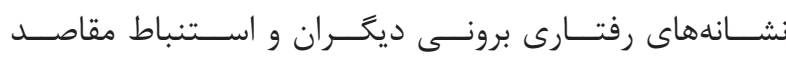

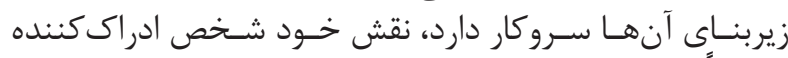

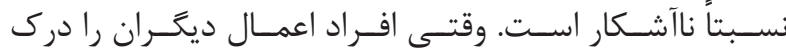

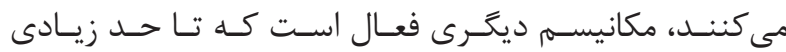

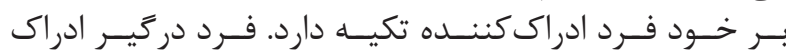

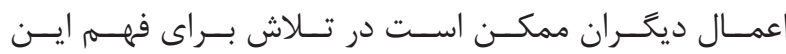

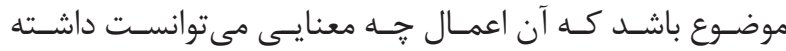

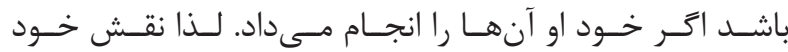

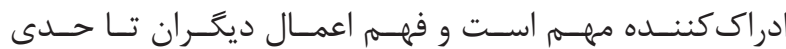

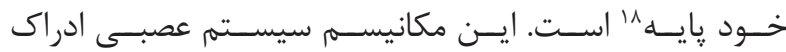

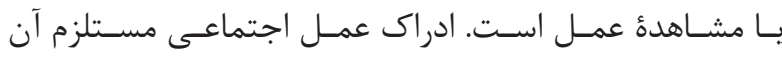

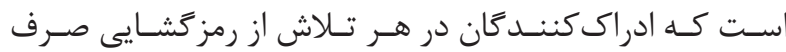

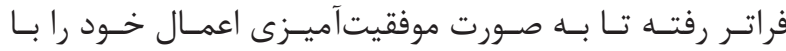

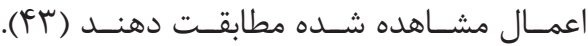

جهــت درك اعمـال ديخـــان، فـرد ادراك كنــــده بايسـد ابتــدا آن

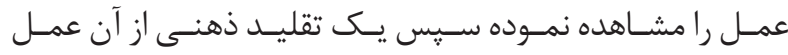

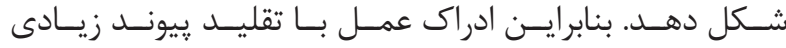

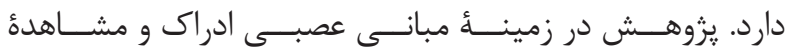

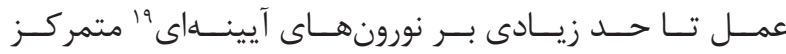

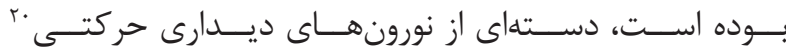

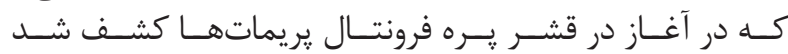

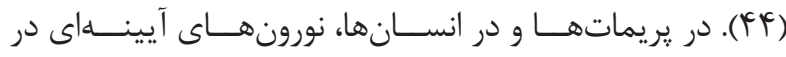

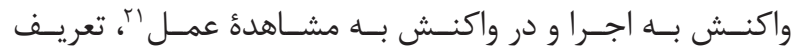

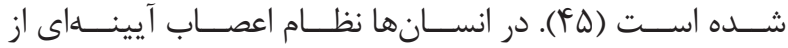

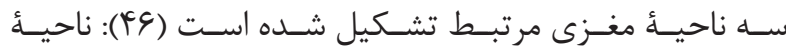

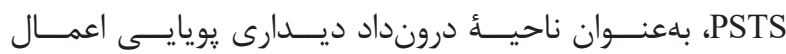

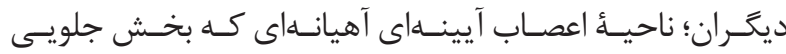

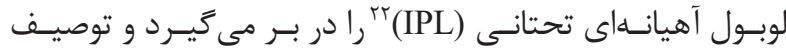

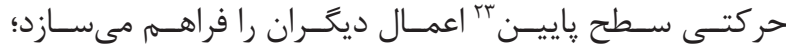

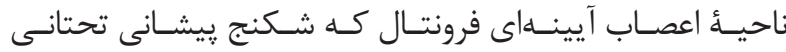
خلفـى (IFG)

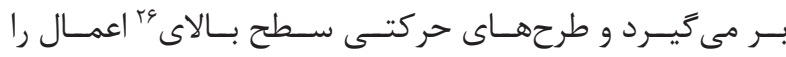

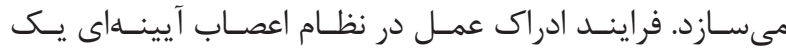

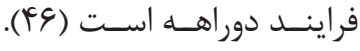

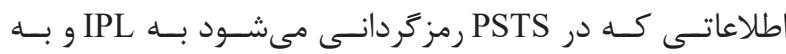

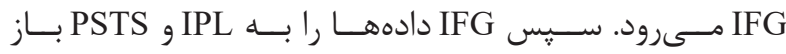

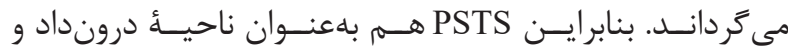

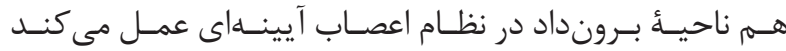
(FV)

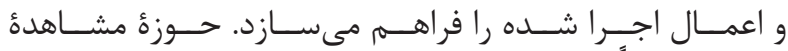

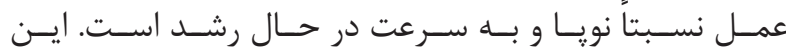

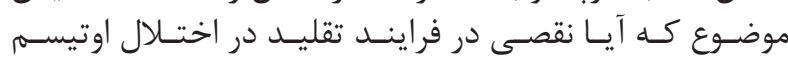

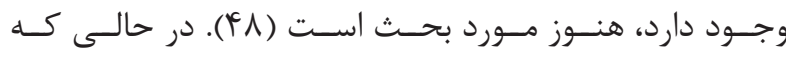

${ }^{14}$ Vocalization

${ }^{15}$ Emotional facial expression

${ }^{16}$ Non vocal sound

${ }^{17}$ Vocal sound

${ }^{18}$ Self-based

${ }^{19}$ Mirror neurons

${ }^{20}$ Visuomotor
مطالعاتـى كـه ادراك اجتماعىى در اختـلالات طيف اوتيسـم را

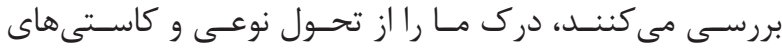

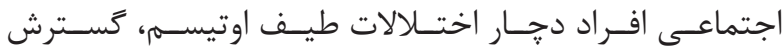

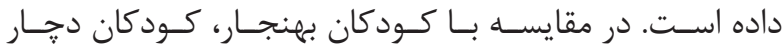

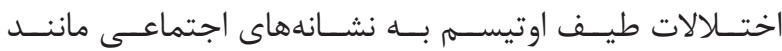

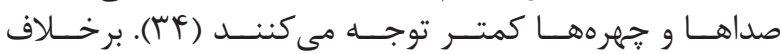

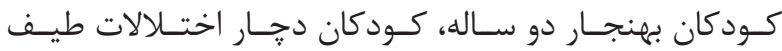

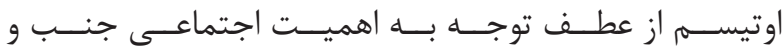

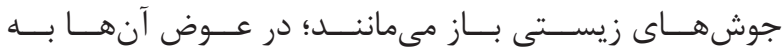

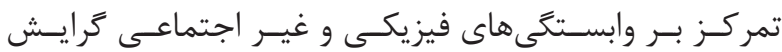

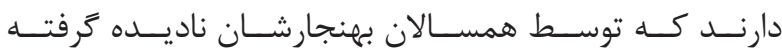

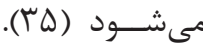

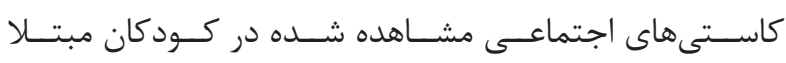

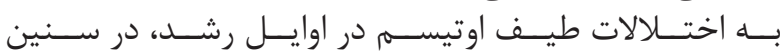

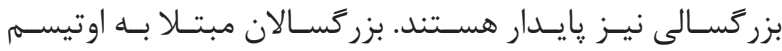

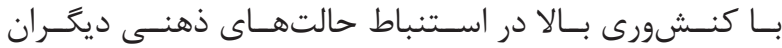

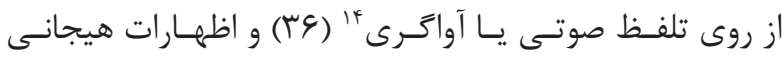

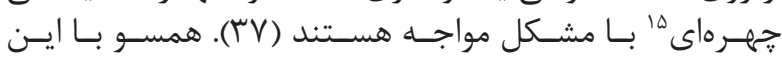

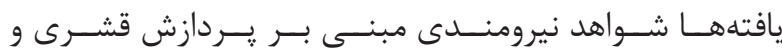

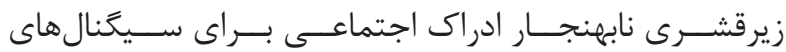

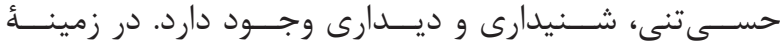

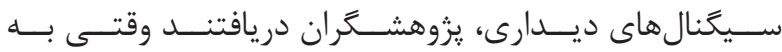

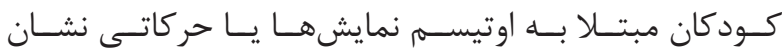

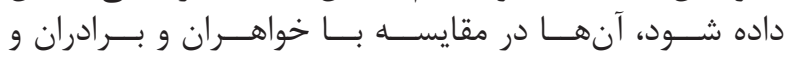

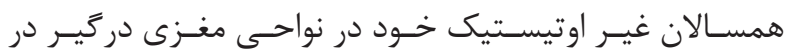

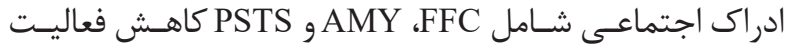

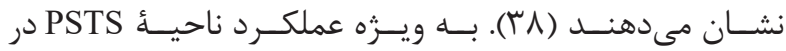

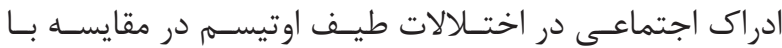

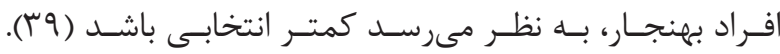

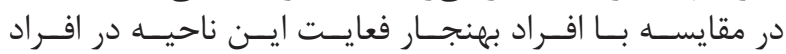

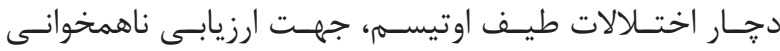

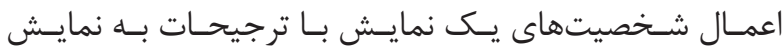

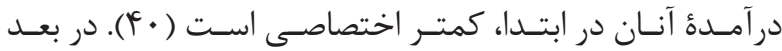

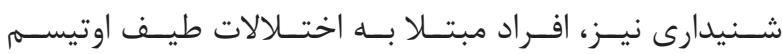

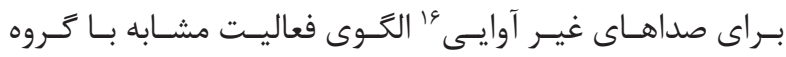

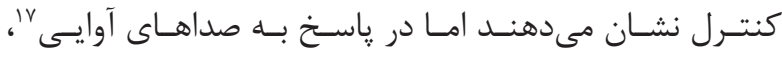

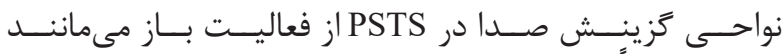

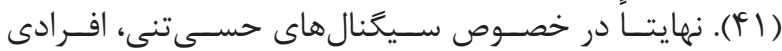

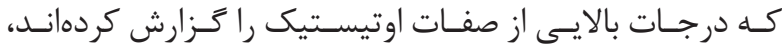

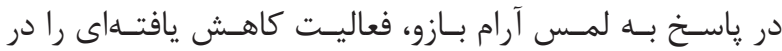

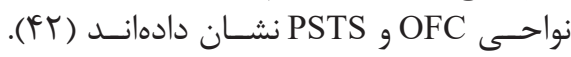
سيستم عصبى مشاهدهُ عمل

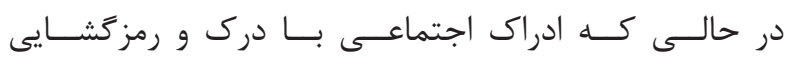

${ }^{21}$ Action observation
${ }^{22}$ Inferior parietal lobule
${ }^{23}$ Lower-level motoric description
${ }^{24}$ Posterior inferior frontal gyrus
${ }^{25}$ Ventral premotor cortex
${ }^{26}$ Higher-level motor plans 
نظـام اعصـاب آيينــهاى همجنـــان ادامــه دارد. سيستم عصبى نظرية ذهن

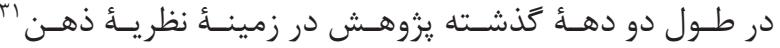

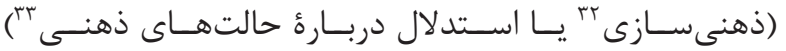

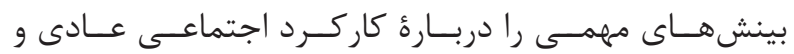

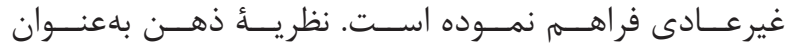

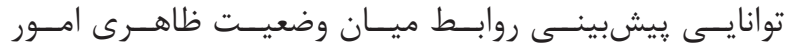

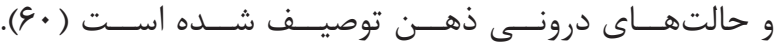

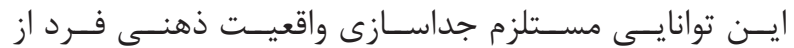

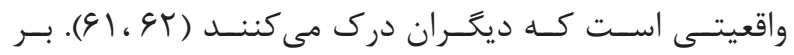

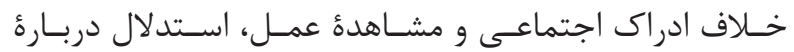

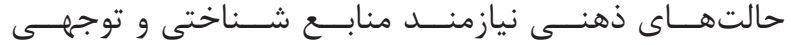

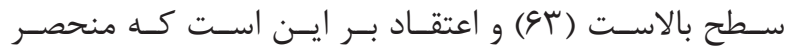

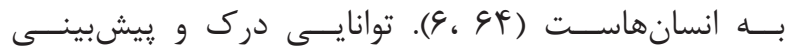

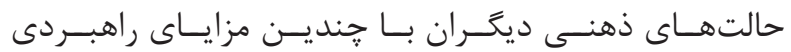

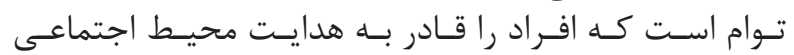

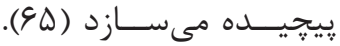

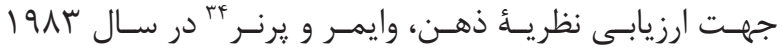

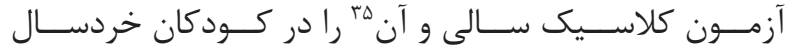

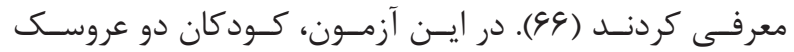

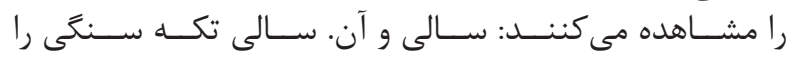

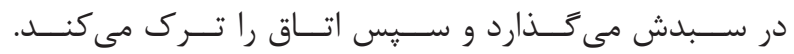

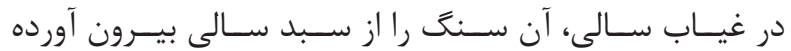

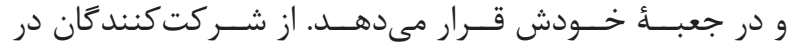

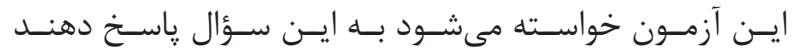

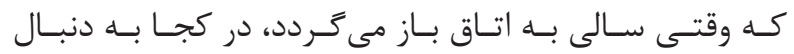

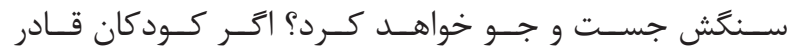

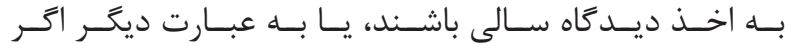

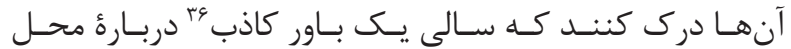

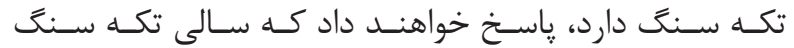

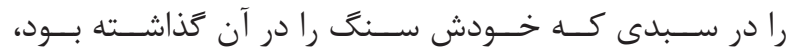

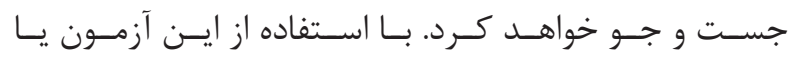

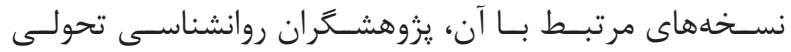

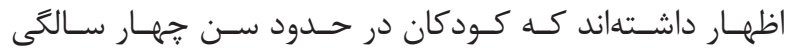

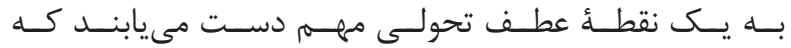

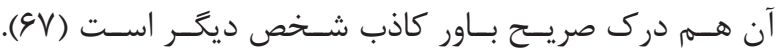

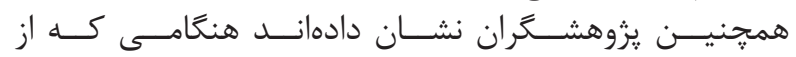

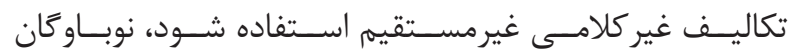

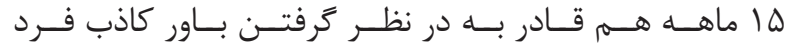

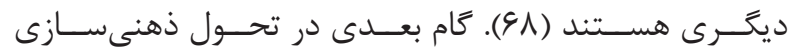

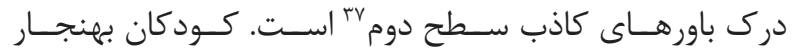

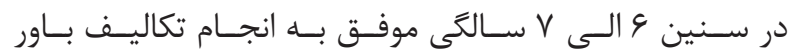

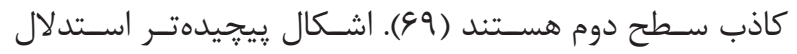

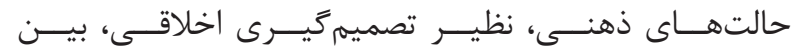

${ }^{27}$ Echolalia

${ }^{28}$ By-product

${ }^{29}$ Functional magnetic resonance imaging

${ }^{30}$ Electroencephalography

${ }^{31}$ Theory of mind

${ }^{32}$ Mentalizing

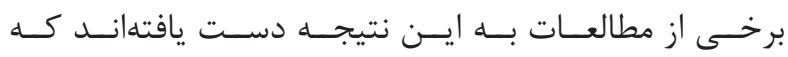

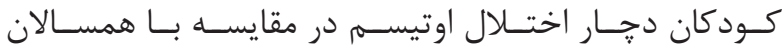

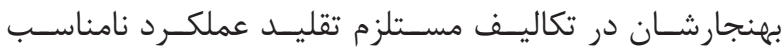

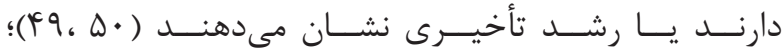

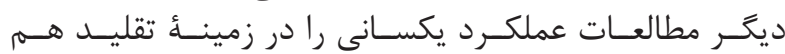

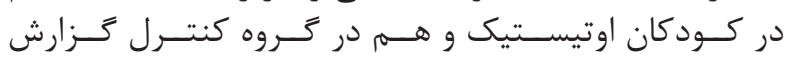

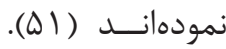

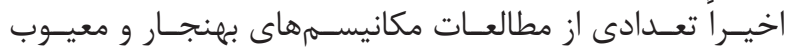

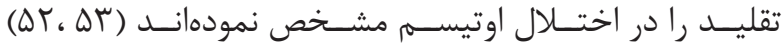

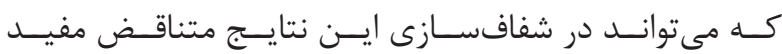

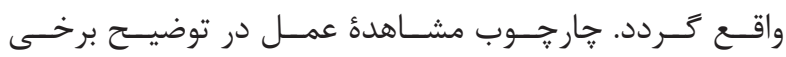

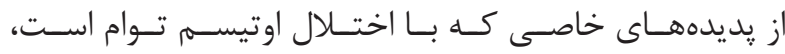

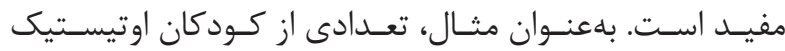

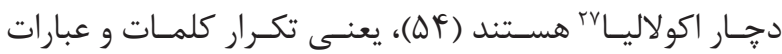

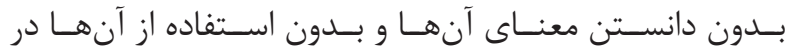

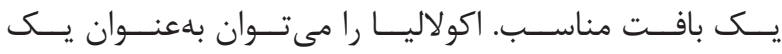

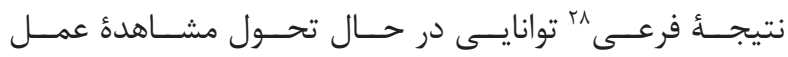

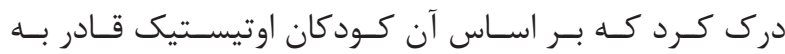

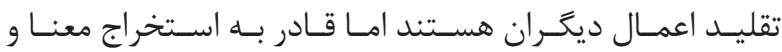

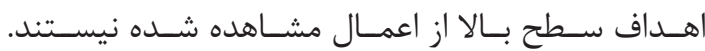
مطالعـات تصويربــردارى عصبـى كـهـ نظـام اعصـاب آيينــاى در

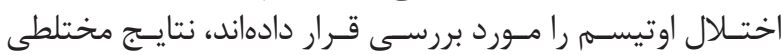

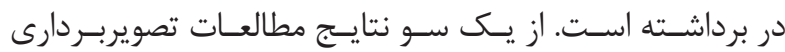

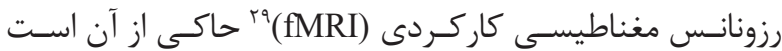

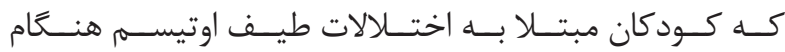

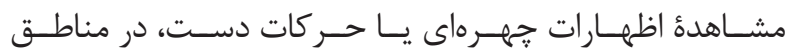

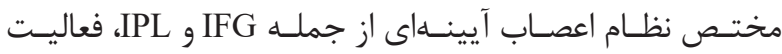

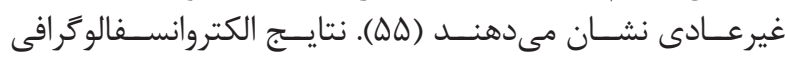

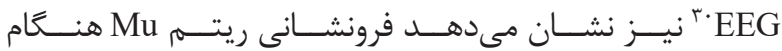

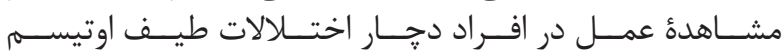

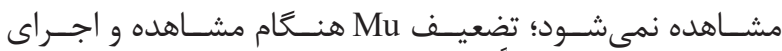

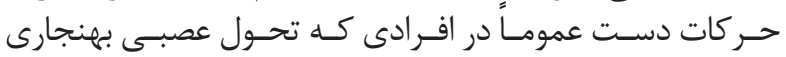

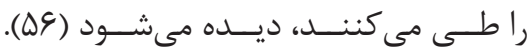

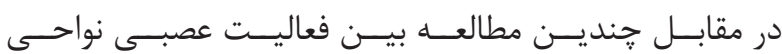

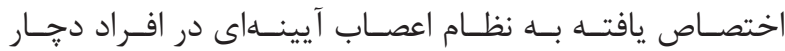

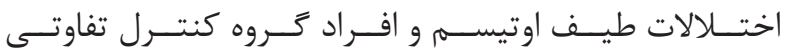

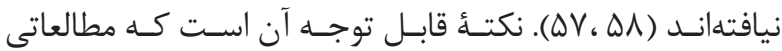

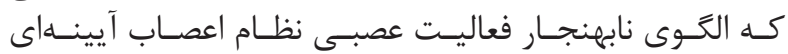

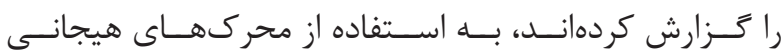

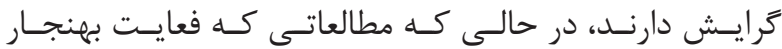

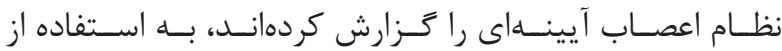

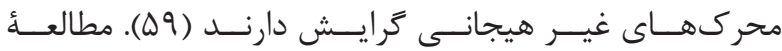

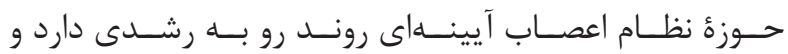

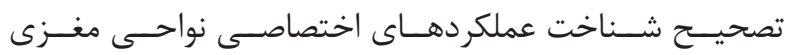

${ }^{33}$ Mental state reasoning

${ }^{34}$ Wimmer and Perner

${ }^{35}$ Classic sally-anne experiment

${ }^{36}$ False belief

${ }^{37}$ Second-order false belief 


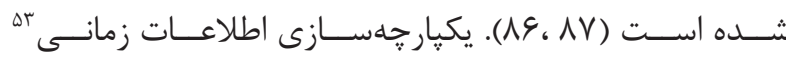

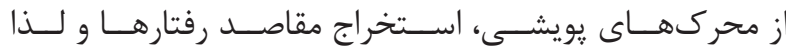

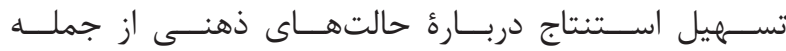

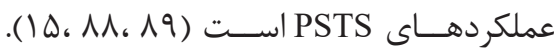

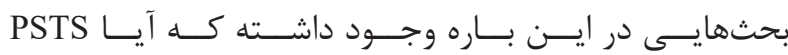

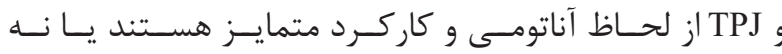

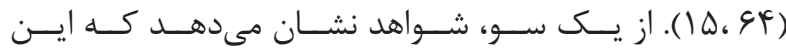

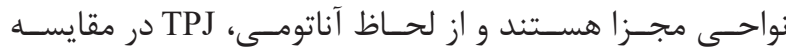

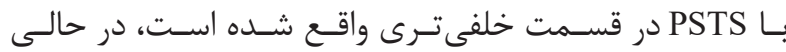

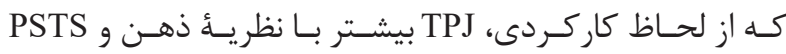

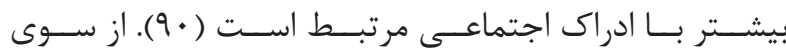

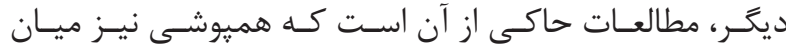

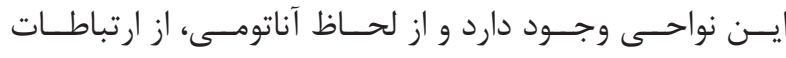

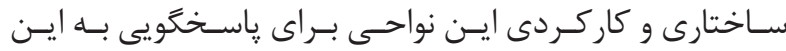

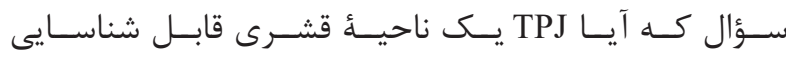

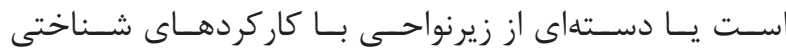

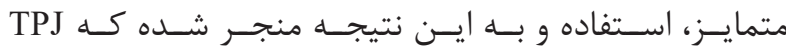

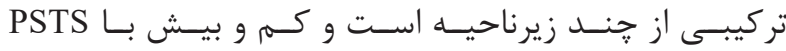

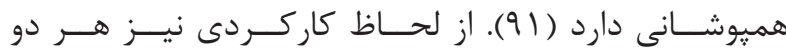

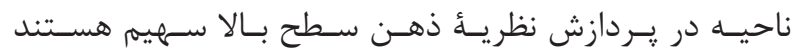
كاركـرد د ، AV)

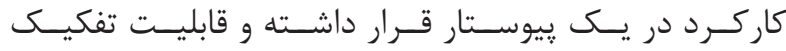

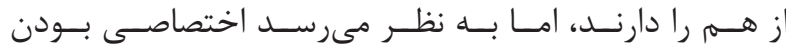

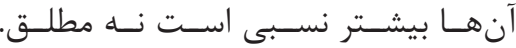

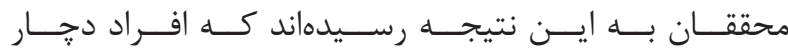

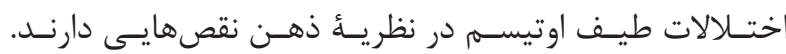

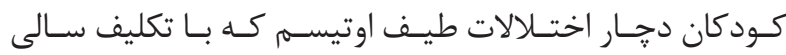

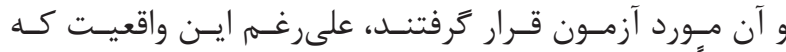

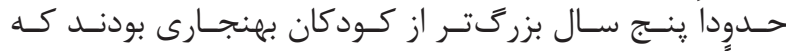

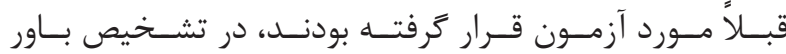

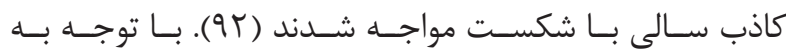

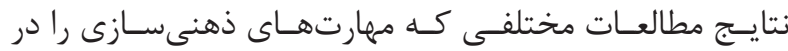

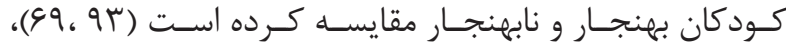

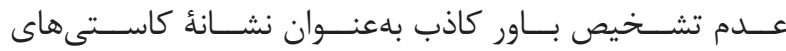

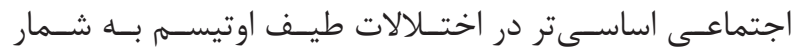

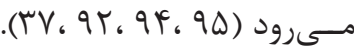

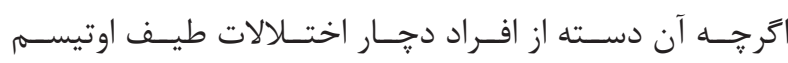

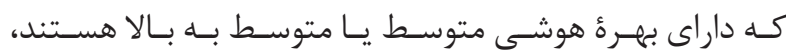

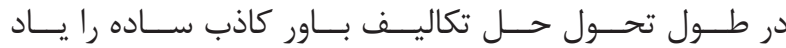

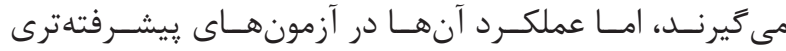

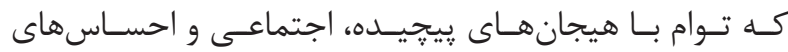

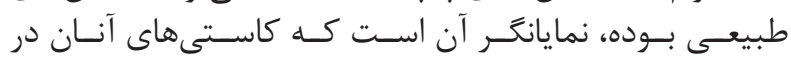

${ }^{38}$ Medial prefrontal cortex

${ }^{39}$ Tempro-parietal Junction

${ }^{40}$ Posterior cingulate cortex/precuneus

${ }^{41}$ Anterior temporal lobes

${ }^{42}$ Temporal poles

${ }^{43}$ Strategic decision-making

${ }^{44}$ Self-other comparisons

${ }^{45}$ Metarepresentation

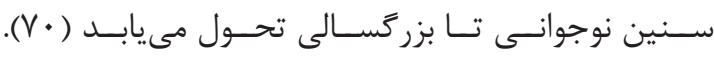

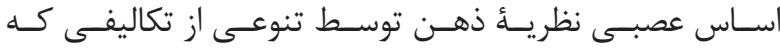

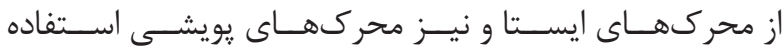

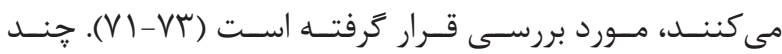

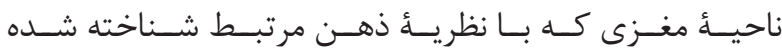

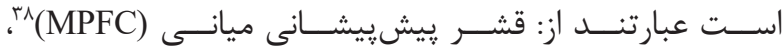

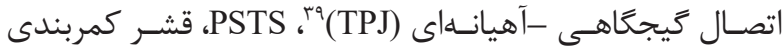
خلفـى / يـره كانئـوس (PCC/PC) (IV، VF، VQ (T) (TP) (ATL) نقـش MPFC در تصميمى

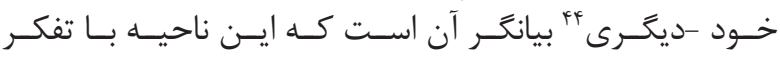

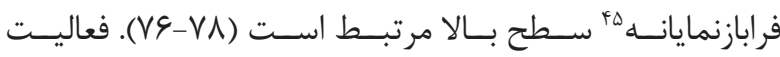

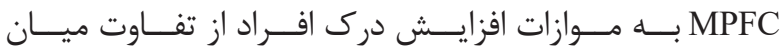

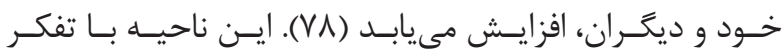

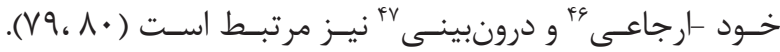

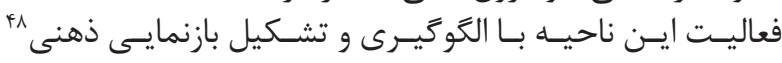

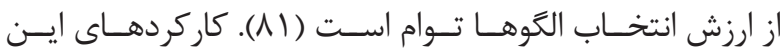

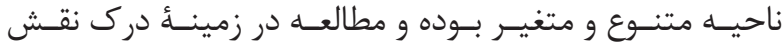

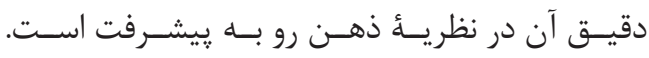

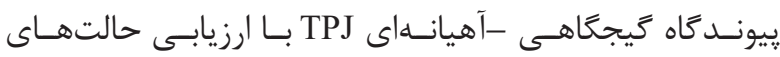

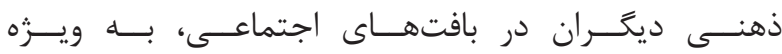

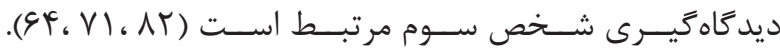

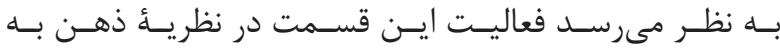

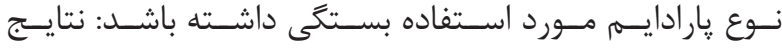

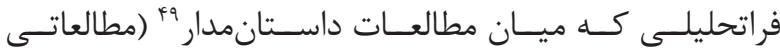

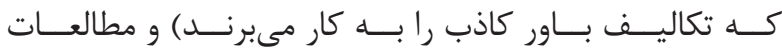

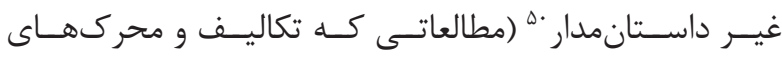

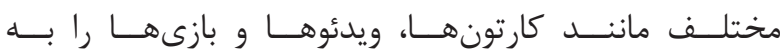

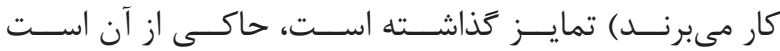

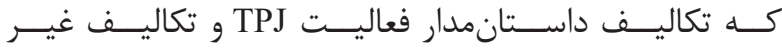

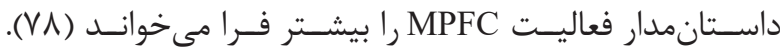

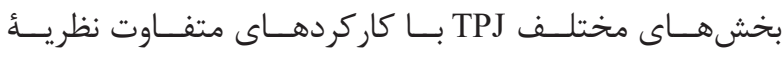

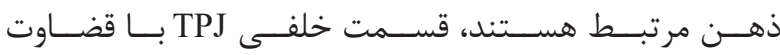

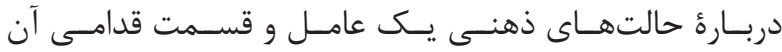

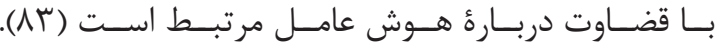

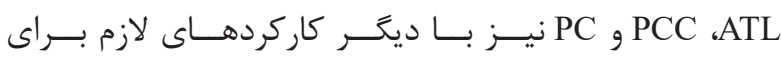

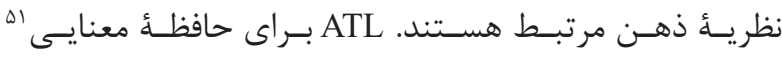

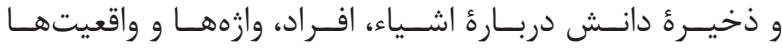

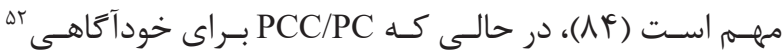

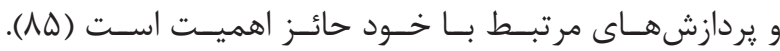

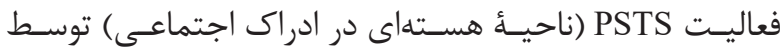

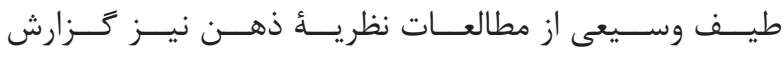

\footnotetext{
${ }^{46}$ Self-referential thinking

${ }^{47}$ Introspection

${ }^{48}$ Mental representation

${ }^{49}$ Story-based

${ }^{50}$ Non-story-based

${ }^{51}$ Semantic memory

${ }^{52}$ Self-awareness

${ }^{53}$ Temporal information
} 
ايـن افـراد در مناطـق مغـزى دخيـل در ادراك اجتماعى شـامل

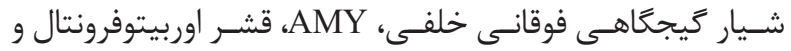

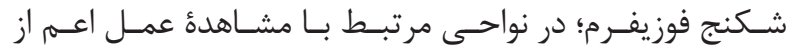

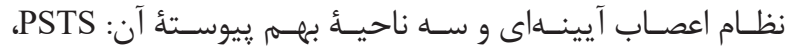

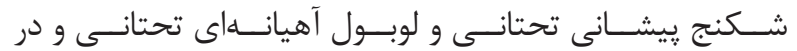

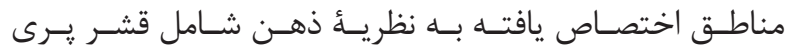

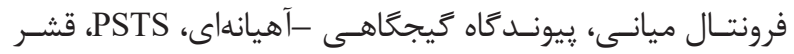

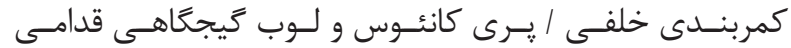

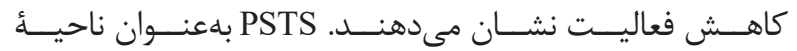

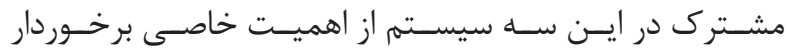

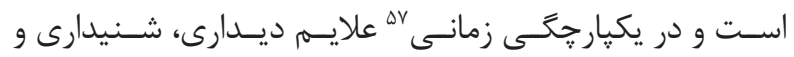

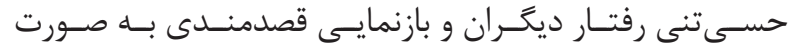

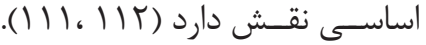

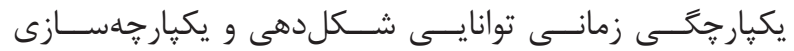

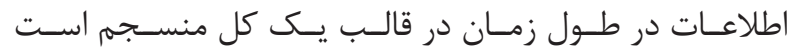

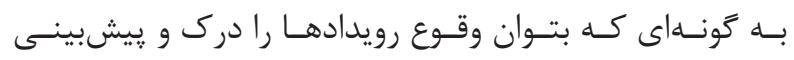

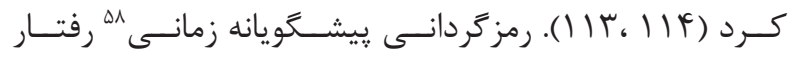

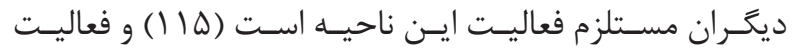

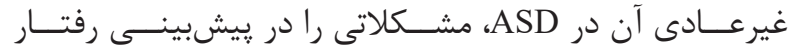

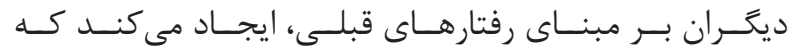

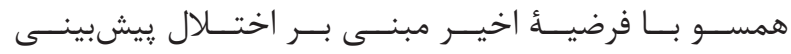

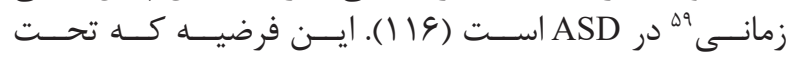

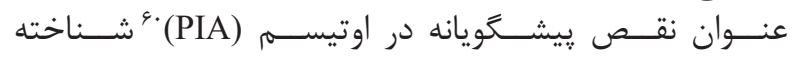

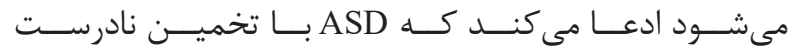

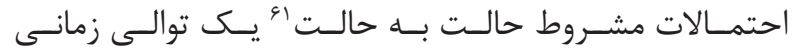

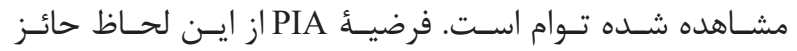

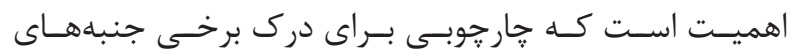

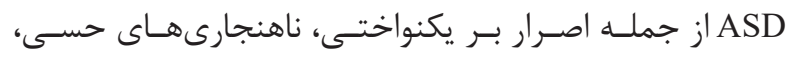

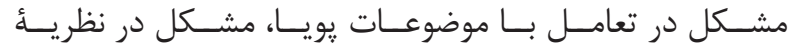

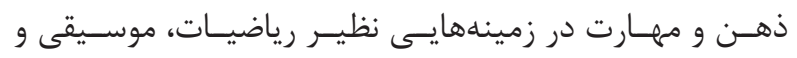

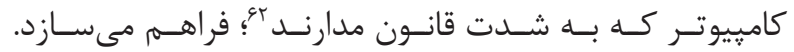

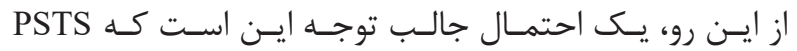

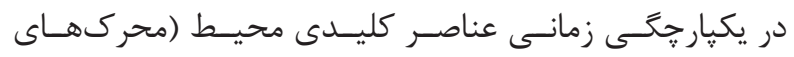

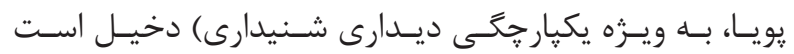

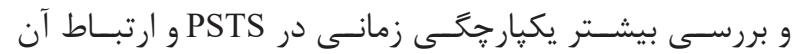

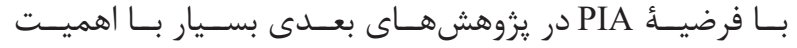

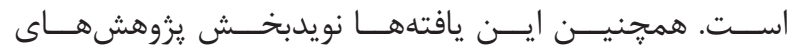

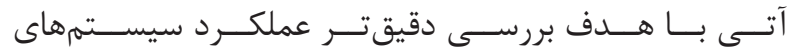

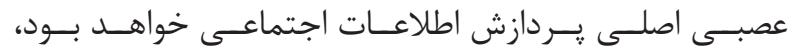

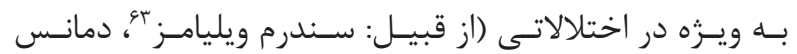

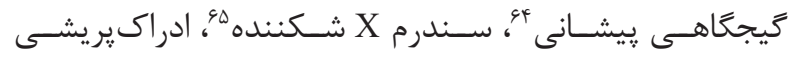

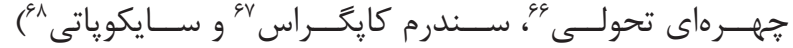

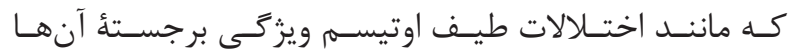

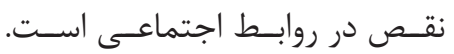

\footnotetext{
${ }^{54}$ Intentional

${ }^{55}$ Unintentional

${ }^{56}$ Goal directed manner

${ }^{57}$ Temporal integration

${ }^{58}$ Temporal predictive coding

${ }^{59}$ Disorder of temporal prediction

${ }^{60}$ Predictive impairment in autism

${ }^{61}$ State to state conditional probabilities
}

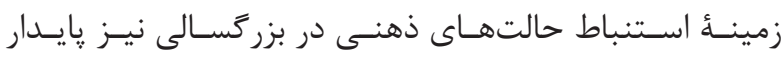

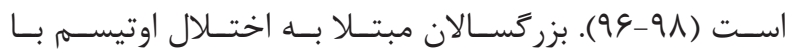

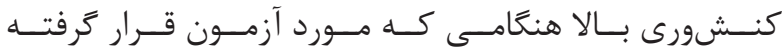

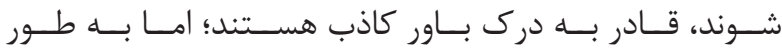

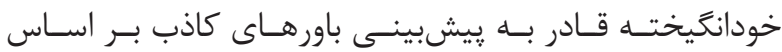
رفتـار نيسـتند (99).

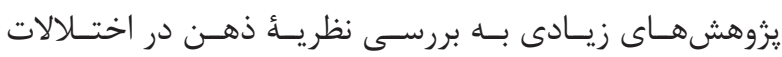

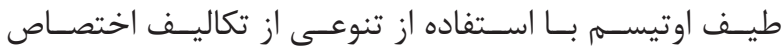

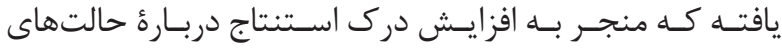

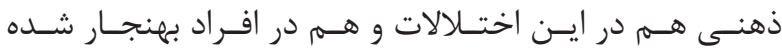

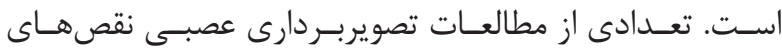

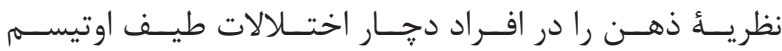

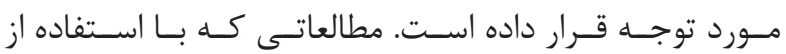

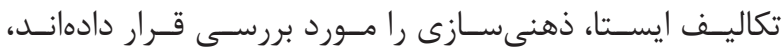

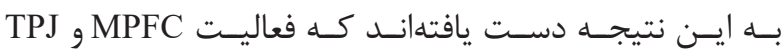

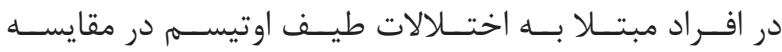

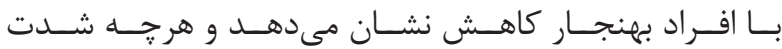

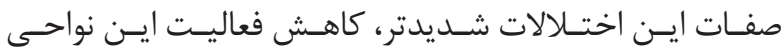

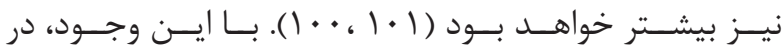

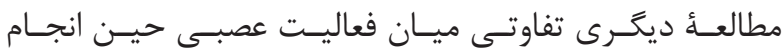

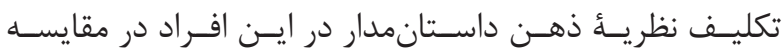

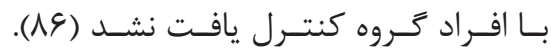

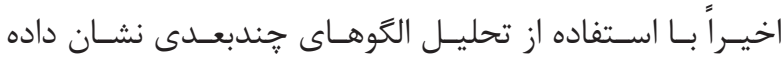

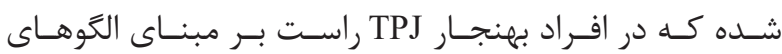

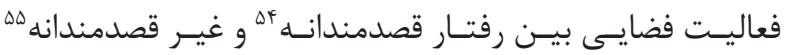

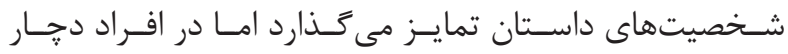

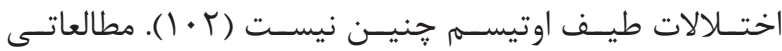

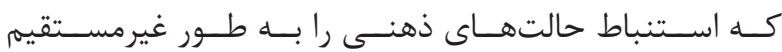

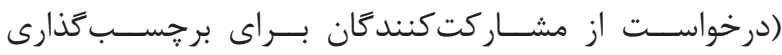

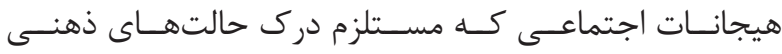

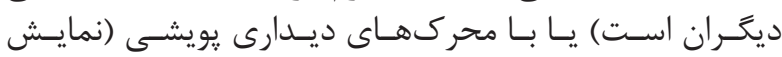

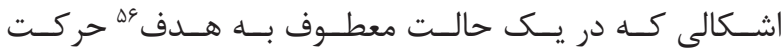

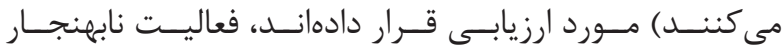

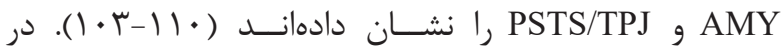

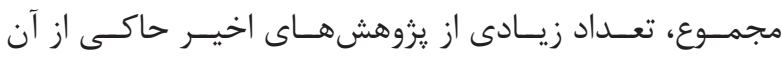

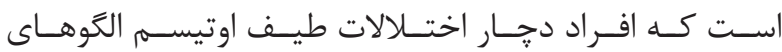

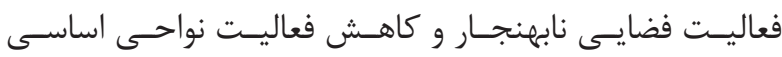

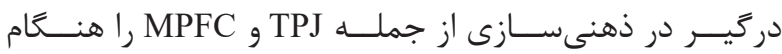

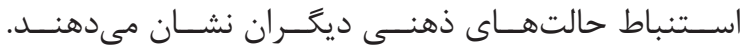

$$
\text { نتيجه }
$$

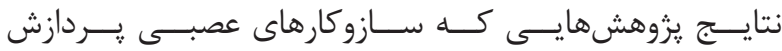

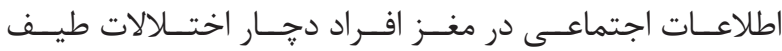

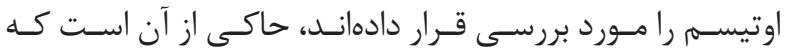
${ }^{62}$ Rule-based
${ }^{63}$ Williams syndrome
${ }^{64}$ Frontotemporal dementia
${ }^{65}$ Fragile $\mathrm{X}$ syndrome
${ }^{66}$ Developmental prosopagnosia
${ }^{67}$ Capgras syndrome
${ }^{68}$ Psychopathy 
1. APA. Diagnostic and statistical manual of mental disorders: DSM-5. $5^{\text {th }}$ ed. American Psychiatric Publishing, Washington DC. 2013. p. 947.

2. Kovacs AM, Teglas E, Endress AD. The social sense: susceptibility to others' beliefs in human infants and adults. Science. 2010; 330(6012): 1830-4.

3. Mcarthur LZ, Baron RM. Toward an ecological theory of social-perception. Psychol Rev. 1983; 90(3): 215-38.

4. Beauchamp MS, Yasar NE, Frye RE, Ro T. Touch, sound and vision in human superior temporal sulcus. Neuroimage. 2008; 41(3): 1011-20.

5. Low J, Perner J. Implicit and explicit theory of mind: state of the art. Br J Dev Psychol. 2012; 30(1): 1-13.

6. Call J, Tomasello M. Does the chimpanzee have a theory of mind? 30 years later. Trends Cogn Sci. 2008; 12(5): 187-92.

7. Emery NJ, Clayton NS. Tool use and physical cognition in birds and mammals. Curr Opin Neurobiol. 2009; 19(1): 27-33.

8. Melis AP, Warneken F, Jensen K, Schneider AC, Call J, Tomasello M. Chimpanzees help conspecifics obtain food and non-food items. Proc Biol Sci. 2011; 278(1710): 1405-13.

9. Flombaum JI, Santos LR. Rhesus monkeys attribute perceptions to others. Curr Biol. 2005; 15(5): 447-52.

10. Santos LR, Nissen AG, Ferrugia JA. Rhesus monkeys, macaca mulatta, know what others can and cannot hear. Anim Behav. 2006; 71(5): 1175-81.

11. Allison T, Puce A, McCarthy G. Social perception from visual cues: role of the STS region. Trends Cogn Sci. 2000; 4(7): 267-78.

12. Ku SP, Tolias AS, Logothetis NK, Goense J. fMRI of the face-processing network in the ventral temporal lobe of awake and anesthetized macaques. Neuron. 2011; 70(2): 352-62.

13. Kreifelts B, Ethofer T, Shiozawa T, Grodd W, Wildgruber D. Cerebral representation of non-verbal emotional perception: fMRI reveals audiovisual integration area between voice- and face-sensitive regions in the superior temporal sulcus. Neuropsychologia. 2009; 47(14): 3059-66.

14. Jastorff J, Popivanov ID, Vogels R, Vanduffel W,
منابع

Orban GA. Integration of shape and motion cues in biological motion processing in the monkey STS. Neuroimage. 2010; 60(2): 911-21.

15. Gobbini MI, Haxby JV. Neural systems for recognition of familiar faces. Neuropsychologia. 2007; 45(1):32-41.

16. Watson R, Latinus M, Charest I, Crabbe F, Belin P. People-selectivity, audiovisual integration and heteromodality in the superior temporal sulcus. Cortex. 2014; 50: 125-36.

17. Bahnemann M, Dziobek I, Prehn K, Wolf I, Heekeren HR. Sociotopy in the temporoparietal cortex: common versus distinct processes. Soc Cogn Affect Neurosci. 2010; 5(1): 48-58

18. Wildgruber D, Ackermann H, Kreifelts B, Ethofer T. Cerebral processing of linguistic and emotional prosody: fMRI studies. Underst Emot. 2006; 156: 249-68.

19. Ethofer T, Anders S, Erb M, Herbert C, Wiethoff $\mathrm{S}$, Kissler J, et al. Cerebral pathways in processing of affectiveprosody: a dynamic causal modeling study. Neuroimage. 2006; 30(2): 580-7.

20. Schwarzlose RF, Baker CI, Kanwisher N. Separate face and body selectivity on the fusiform gyrus. J Neurosci. 2005; 25(47): 11055-9.

21. Morris JP, Pelphrey KA, McCarthy G. Controlled scanpath variation alters fusiform face activation. Soc Cogn Affect Neurosci. 2007a; 2(1): 31-8.

22. Morris JP, Pelphrey KA, McCarthy G. Face processing without aware-ness in the right fusiform gyrus. Neuropsychologia. 2007b; 45(13): 3087-91.

23. Pitcher D, Dilks DD, Saxe RR, Triantafyllou C, Kanwisher N. Dif-ferential selectivity for dynamic versus static information in face-selective cortical regions. Neuroimage. 2011; 56(4): 2356-63.

24. Gobbini MI, Gentili C, Ricciardi E, Bellucci C, Salvini $\mathrm{P}$, Laschi C, et al. Distinct neural systems involved in agency and ani-macy detection. J Cogn Neurosci. 2011; 23(8): 1911-20.

25. Shultz S, McCarthy G. Goal-directed actions activate the face-sensitive posterior superior temporal sulcus and fusiform gyrus in the absence of human-like perceptual cues. Cereb Cortex. 2012; 22(5): 1098-106.

26. Costafreda SG, Brammer MJ, David AS, Fu CHY. Predictors of amyg-dala activation during the processing of emotional stimuli: a meta-analysisof 385 PET and 
fMRI studies. Brain Res. Rev. 2008; 58(1): 57-70.

27. Adolphs R. The social brain: neural basis of social knowledge. Annu Rev Psychol. 2009; 60: 693-716.

28. Adolphs R, Gosselin F, Buchanan TW, Tranel D, Schyns P, Damasio AR. A mechanism for impaired fear recognition after amygdala damage. Nature. 2005; 433(7021): $68-72$.

29. Adolphs R. Fear, faces, and the human amygdala. Curr Opin Neurobiol. 2008; 18(2): 166-72.

30. Watson KK, Platt ML. Social signals in primate orbitofrontal cortex. Curr Biol. 2012; 22(23): 2268-73.

31. Ethofer T, Bretscher J, Wiethoff S, Bisch J, Schlipf S, Wildgruber D, et al. Functional responses and structural connections of cortical areas for processing faces and voices in the superior temporal sulcus. Neuroimage. 2013; 76(1): 45-56.

32. Kahnt T, Heinzle J, Park SQ, Haynes JD. The neural code of reward antic-ipation in human orbitofrontal cortex. Proc Natl Acad Sci USA. 2010; 107(13): 6010-5.

33. Wallis JD. Cross-species studies of orbitofrontal cortex and value-baseddecision-making. Nat Neurosci. 2012; 15(1): 13-9.

34. Chawarska K, Macari S, Shic F. Decreased spontaneous attention to social scenes in 6-month-old infants later diagnosed with autism spectrum disorders. Biol Psychiatry. 2013; 74(3): 195-203.

35. Klin A, Lin DJ, Gorrindo P, Ramsay G, Jones W. Two-year-olds with autism orient to non-social contingencies rather than biological motion. Nature. 2009; 459(7244): 257-61.

36. Rutherford MD, Baron-Cohen S, Wheelwright S. Reading the mind in the voice: a study with normal adults and adults with Asperger syndrome and high functioning autism. J Autism Dev Disord. 2002; 32(3): 189-94.

37. Baron-Cohen S, Wheelwright S, Hill J, Raste Y, Plumb I. The "reading the mind in the eyes" test revised version: a study with normal adults, and adults with asperger syndrome or high-functioning autism. J Child Psychol Psychiatry. 2001; 42(2): 241-51.

38. Kaiser MD, Hudac CM, Shultz S, Lee SM, Cheung C, Berken AM, et al. Neural signatures of autism. Proc Natl Acad Sci USA. 2010; 107(49): 21223-8.

39. Pelphrey KA, Morris JP, McCarthy G. Neural basis of eye gaze processing deficits in autism. Brain. 2005;
128: $1038-48$.

40. Vander Wyk B, Hudac CM, Carter EJ, Sobel DM, Pelphrey KA. Action understanding in the superior temporal sulcus region. Psychol Sci. 2009; 20(6): 771-7.

41. Gervais H, Belin P, Boddaert N, Leboyer M, Coez A, Sfaello I, et al. Abnormal cortical voice processing in autism. Nat Neurosci. 2004; 7(8): 801-2.

42. Voos AC, Pelphrey KA, Kaiser MD. Autistic traits are associated with diminished neural response to affective touch. Soc Cogn Affect Neurosci. 2013; 8(4): 378-86.

43. Williams JHG, Whiten A, Singh T. A systematic review of action imitation in autistic spectrum disorder. J Autism Dev Disord. 2004; 34(3): 285-99.

44. Matelli M, Luppino G, Rizzolatti G. Patterns of cytochrome-oxidase activity in the frontal agranular cortex of the macaque monkey. Behav Brain Res. 1985; 18(2): $125-36$.

45. Keysers C, Gazzola V. Social neuroscience: mirror neurons recorded in humans. Curr Biol. 2010; 20(8): 353-4.

46. Iacoboni M, Dapretto M. The mirror neuronsys tem and the consequences of its dysfunction. Nat Rev Neurosci. 2006; 7(12): 942-51.

47. Rizzolatti G, Cattaneo L, Fabbri-Des tro M, Rozzi S. Cortical mechanisms underlying the organization of goal-directed actions and mirror neuron based action understanding. Physiol Rev 2014; 94(2): 655-706.

48. Sevlever M, Gillis JM. An examination of the state of imitation research in children with autism: issues of definition and methodology. Res Dev Disabil. 2010; 31(5): 976-84.

49. Stewart HJ, McIntosh RD, Williams JHG. A specific deficit of imitation in autism spectrum disorder. Autism Res. 2013; 6(6): 522-30.

50. Young GS, Rogers SJ, Hutman T, Rozga A, Sigman M, Ozonoff S. Imitation from 12 to 24 months in autism and typical development: a longitudinal Rasch analysis. Dev Psychol. 2011; 47(6): 1565-78.

51. Press C, Richardson D, Bird G. Intact imitation of emotional facial actions in autism spectrum conditions. Neuropsychologia. 2010; 48(11): 3291-7.

52. Vivanti G, McCormick C, Young GS, Abucayan F, Hatt N, Nadig A, et al. Intact and impaired mechanisms 
of action understanding in autism. Dev Psychol. 2011; 47(3): 841-56.

53. Vivanti G, Trembath D, Dissanayake C. Mechanisms of imitation impairment in autism spectrum disorder. J. Abnorm. Child Psychol. 2014. http://dx.doi.org/10.1007/ s10802-014-9874-9.

54. Prizant BM, Duchan JF. The functions of immediate echolalia in autistic children. J Speech Hear Disord. 1981; 46(3): 241-9.

55. Martineau J, Andersson F, Barthelemy C, Cottier JP, Destrieux C. Atypical activation of the mirror neuron system during perception of hand motion in autism. Brain Res. 2010; 1320: 168-75.

56. Oberman LM, McCleery JP, Hubbard EM, Bernier R, Wiersema JR, Raymaekers R, et al. Developmental changes in mu suppression to observed and executed actions in autism spectrum disorders. Soc Cogn Affect Neurosci. 2013; 8(3): 300-4.

57. Bernier R, Aaronson B, McPartland J. The role of imitation in the observed heterogeneity in EEG mu rhythm in autism and typical development. Brain Cogn. 2013; 82(1): 69-75.

58. Dinstein I, Thomas C, Humphreys K, Minshew N, Behrmann M, Heeger DJ. Normal movement selectivity in autism. Neuron. 2010; 66(3): 461-9.

59. Hamilton AFD. Reflecting on the mirror neuron sys tem in autism: a systematic review of current theories. Dev Cogn Neurosci. 2013; 3: 91-105.

60. Frith U. Autism: explaining the enigma. Basil Blackwell, Cambridge, MA. 1989.

61. Baillargeon R, Scott RM, He Z. False-belief unders tanding in infants. Trends Cogn Sci. 2010; 14(3): 110-8.

62. Blakemore SJ, den Ouden H, Choudhury S, Frith C. Adolescent development of the neural circuitry for thinking about intentions. Soc Cogn Affect Neurosci. 2007; 2(2): 130-9.

63. Lin SH, Keysar B, Epley N. Reflexively mind blind: using theory of mind to interpret behavior requires effortful attention. J Exp Soc Psychol. 2010; 46(3): 551-6.

64. Saxe R. Uniquely human social cognition. Curr Opin Neurobiol. 2006; 16(2): 235-9.

65. Wan C. Shared knowledge matters: culture as intersubjective representations. Soc Personal Psychol Compass 2012; 6(2): 109-25.
66. Wimmer H, Perner J. Beliefs about beliefs: representation and constraining function of wrong beliefs in young children's understanding of deception. Cognition. 1983; 13(1): 103-28.

67. Perner J, Mauer MC, Hildenbrand M. Identity: key to children's understanding of belief. Science. 2011; 333(6041): 474-7.

68. Onishi KH, Baillargeon R. Do 15-month-old infants understand false beliefs? Science. 2005; 308(5719): 255-8.

69. Perner J, Wimmer H. John thinks that Mary thinks that - attribution of 2 ndorder beliefs by 5 -year-old to 10-year-old children. J Exp Child Psychol. 1985; 39: 437-71.

70. Sebastian CL, Fontaine NMG, Bird G, Blakemore SJ, De Brito SA, McCrory EJP, et al. Neural processing associated with cognitive and affective theory of mind in adolescents and adults. Soc Cogn Affect Neurosci. 2012; 7(1): 53-63.

71. Saxe R, Kanwisher N. People thinking about thinking people. the role of the temporo-parietal junction in "theory of mind". Neuroimage. 2003; 19(4): 1835-42.

72. Walter H, Adenzato M, Ciaramidaro A, Enrici I, Pia L, Bara BG. Understanding intentions in social interaction: the role of the anterior paracingulate cortex. J Cogn Neurosci. 2004; 16(10): 1854-63.

73. Wolf I, Dziobek I, Heekeren HR. Neural correlates of social cognition in naturalistic settings: a model-free analysis approach. Neuroimage. 2010; 49(1): 894-904.

74. Amodio DM, Frith CD. Meeting of minds: the medial frontal cortex and social cognition. Nat Rev Neurosci. 2006; 7(4): 268-77.

75. Mar RA. The neural bases of social cognition and $\mathrm{s}$ tory comprehension. Annu Rev Psychol. 2011; 62: 103 34.

76. Coricelli G, Nagel R. Neural correlates of depth of s trategic reasoning in medial prefrontal cortex. Proc Natl Acad Sci USA. 2009; 106(23): 9163-8.

77. Lombardo MV, Baron-Cohen S. Unraveling the paradox of the autistic self. Wiley Interdiscip. Rev Cogn Sci. 2010; 1(3): 393-403.

78. Tamir DI, Mitchell JP. Neural correlates of anchoringand-adjustment during mentalizing. Proc Natl Acad Sci USA. 2010; 107(24): 10827-32.

79. Ochsner KN, Knierim K, Ludlow DH, Hanelin J, 


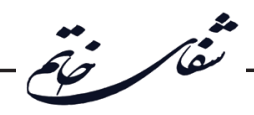

Ramachandran T, Glover G, et al. Reflecting upon feelings: an fMRI study of neural systems supporting the attribution of emotion to self and other. J Cogn Neurosci. 2004; 16(10): 1746-72.

80. Silani G, Bird G, Brindley R, Singer T, Frith C, Frith U. Levels of emotional awareness and autism: an fMRI study. Soc Neurosci. 2008; 3(2): 97-112.

81. Nicolle A, Klein-Flugge MC, Hunt LT, Vlaev I, Dolan RJ, Behrens TEJ. An agent independent axis for executed and modeled choice in medial prefrontal cortex. Neuron. 2013; 75(6): 1114-21.

82. Carter RM, Bowling DL, Reeck C, Huettel SA. A dis tinct role of the temporal-parietal junction in predicting socially guided decisions. Science. 2012; 337(6090): 109-11.

83. Takahashi H, Terada K, Morita T, Suzuki S, Haji T, Kozima H, et al. Different impressions of other agents obtained through social interaction uniquely modulate dorsal and ventral pathway activities in the social human brain. Cortex. 2014; 58: 289-300.

84. Bonner MF, Price AR. Where is the anterior temporal lobe and what does it do? J Neurosci. 2013; 33(10): 4213-5.

85. den Ouden HEM, Frith U, Frith C, Blakemore SJ. Thinking about intentions. Neuroimage. 2005; 28(4): 787-96.

86. Dufour N, Redcay E, Young L, Mavros PL, Moran JM, Triantafyllou C, et al. Similar brain activation during false belief tasks in a large sample of adults with and without autism. Plos One. 2013; 8(9): 754-68.

87. Walter H, Schnell K, Erk S, Arnold C, Kirsch P, Esslinger C, et al. Effects of a genome-wide supported psychosis risk variant on neural activation during a theory-of-mind task. Mol Psychiatry. 2011; 16(4): 46270 .

88. Barch DM, Burgess GC, Harms MP, Petersen SE, Schlaggar BL, Corbetta M, et al. Function in the human connectome: task-fMRI and individual differences in behavior. Neuroimage. 2013; 80: 169-89.

89. Vanderwal T, Hunyadi E, Grupe DW, Connors CM, Schultz RT. Self, mother and abstract other: an fMRI study of reflective social processing. Neuroimage. 2008; 41(4): 1437-46.

90. Saxe R, Whitfield-Gabrieli S, Scholz J, Pelphrey KA. Brain regions for perceiving and reasoning about other people in school-aged children. Child Dev. 2009; 80(4): 1197-209.
91. Mars RB, Sallet J, Schuffelgen U, Jbabdi S, Toni I, Rushworth MFS. Connectivity-based subdivisions of the human right "temporoparietal junction area": evidence for different areas participating in different cortical networks. Cereb Cortex. 2012; 22(8): 1894-903.

92. Baron-Cohen S, Leslie AM, Frith U. Does the autis tic child have a "theory of mind"? Cognition. 1985; 21(1): 37-46.

93. Baron-Cohen S. The autistic child's theory of mind: a case of specific developmental delay. J Child Psychol. Psychiatry. 1989; 30(2): 285-97.

94. Baron-Cohen S, Jolliffe T, Mortimore C, Robertson M. Another advanced test of theory of mind: evidence from very high functioning adults with autism or asperger syndrome. J Child Psychol Psychiatry. 1997; 38(7): 813-22.

95. Leslie AM, Thaiss L. Domain specificity in conceptual development: neuropsychological evidence from autism. Cognition. 1992; 43(3): 225-51.

96. Happé FG. An advanced test of theory of mind: understanding of story characters' thoughts and feelings by able autistic, mentally handicapped, and normal children and adults. J Autism Dev Disord. 1994; 24(2): 129-54.

97. Moran JM, Young LL, Saxe R, Lee SM, O’Young $\mathrm{D}$, Mavros PL, et al. Impaired theory of mind for moral judgment in high-functioning autism. Proc Natl Acad Sci USA. 2011; 108(7): 2688-92.

98. Rosenblau G, Kliemann D, Heekeren H, Dziobek I. Approximating implicit and explicit mentalizing with two naturalistic video-based tasks in typical development and autism spectrum disorder. J Autism Dev Disord. 2015; 45(4): 953-65.

99. Senju A, Southgate V, White S, Frith U. Mindblind eyes: an absence of spontaneous theory of mind in asperger syndrome. Science. 2009; 325(5942): 883-5.

100. Lombardo MV, Chakrabarti B, Bullmore ET, Baron-Cohen S, Consortium MA. Specialization of right temporo-parietal junction for mentalizing and its relation to social impairments in autism. Neuroimage. 2011; 56(3): 1832-8.

101. Happé F, Ehlers S, Fletcher P, Frith U, Johansson $\mathrm{M}$, Gillberg C, et al. 'Theory of mind' in the brain. evidence from a PET scan study of asperger syndrome. Neuroreport. 1996; 8(1): 197-201.

102. Koster-Hale J, Saxe R, Dungan J, Young LL. 
Decoding moral judgments from neural representations of intentions. Proc Natl Acad Sci USA. 2013; 110(14): 5648-53.

103. Baron-Cohen S, Ring HA, Wheelwright S, Bullmore ET, Brammer MJ, Simmons A, et al. Social intelligence in the normal and autistic brain: an fMRI s tudy. Eur J Neurosci. 1999; 11(6): 1891-8.

104. Critchley HD, Daly EM, Bullmore ET, Williams SC, Van Amelsvoort T, Robertson DM, et al. The functional neuroanatomy of social behaviour: changes in cerebral blood flow when people with autis tic disorder process facial expressions. Brain. 2000; 123(11): 2203-12.

105. Castelli F, Frith C, Happé F, Frith U. Autism, asperger syndrome and brain mechanisms for the attribution of mental states to animated shapes. Brain. 2002; 125(8): 1839-49.

106. Pelphrey KA, Shultz S, Hudac CM, Vander Wyk BC. Research review: constraining heterogeneity: the social brain and its development in autism spectrum disorder. J Child Psychol Psychiatry. 2011; 52(6): 63144.

107. Perlman SB, Hudac CM, Pegors T, Minshew NJ, Pelphrey KA. Experimental manipulation of faceevoked activity in the fusiform gyrus of individuals with autism. Soc Neurosci. 2011; 6(1): 22-30.

108. Kliemann D, Dziobek I, Hatri A, Baudewig J, Heekeren HR. The role of the amygdala in atypical gaze on emotional faces in autism spectrum disorders. $\mathrm{J}$ Neurosci. 2012; 32(28): 9469-76.

109. Cheng W, Rolls ET, Gu H, Zang J, Feng G. Autism: reduced connectivity between cortical areas involved in face expression, theory of mind, and the sense of self. Brain. 2015; 138(5): 1382-93.

110. O’Nions E, Sebastian CL, McCrory E, Chantiluke K, Happé F, Viding E. Neural bases of theory of mind in children with autism spectrum disorders and children with conduct problems and callous-unemotional traits. Dev Sci. 2014; 17(5): 786-96.

111. Gordon I, Voos AC, Bennett RH, Bolling DZ, Pelphrey KA, Kaiser MD. Brain mechanisms for processing affective touch. Hum Brain Mapp. 2013; 34(4): $914-22$.

112. Hagan CC, Woods W, Johnson S, Green GGR, Young AW. Involvement of right STS in audio-visual integration for affective speech demonstrated using MEG. Plos One. 2013; 8(8): 1-12.

113. Nakano T, Ota H, Kato N, Kitazawa S. Deficit in visual temporal integration in autism spectrum disorders. Proc Biol Sci. 2010; 277(1684): 1027-30.

114. Stevenson RA, Siemann JK, Schneider BC, Eberly HE, Woynaroski TG, Camarata SM, et al. Multisensory temporal integration in autism spectrum disorders. J Neurosci. 2014; 34(3): 691-7.

115. c H, Friston KJ, Blakemore SJ. Effective connectivity during animacy perception - dynamic causal modelling of human connectome project data. Sci Rep. 2014; 4: 6240. doi: $10.1038 /$ srep06240.

116. Sinha P, Kjelgaard MM, Gandhi TK, Tsourides $\mathrm{K}$, Cardinaux AL, Pantazis D. Autism as a disorder of prediction. Proc Natl Acad Sci USA. 2014; 111(42): 15220-5. 